\title{
Effectiveness of Ceftriaxone Plus Doxycycline in the Treatment of Patients Hospitalized with Community- Acquired Pneumonia
}

\author{
Scott A. Flanders, MD $^{1}$ \\ Vicky Dudas, Pharmo ${ }^{2}$ \\ Kathleen Kerr, $\mathrm{BA}^{3}$ \\ Charles E. McCulloch, PhD ${ }^{4}$ \\ Ralph Gonzales, MD, MSPH ${ }^{3,4,5}$ \\ ${ }^{1}$ Department of Medicine, University of Michigan,
Ann Arbor, Michigan \\ ${ }^{2}$ Department of Clinical Pharmacy, School of \\ Pharmacy, University of California, San Francisco, \\ San Francisco, California \\ ${ }^{3}$ Department of Medicine, University of California, \\ San Francisco, San Francisco, California \\ ${ }^{4}$ Department of Epidemiology and Biostatistics, \\ University of California, San Francisco, San Fran- \\ cisco, California \\ ${ }^{5}$ Division of General Internal Medicine, University \\ of California, San Francisco, San Francisco, Cali- \\ fornia
}

BACKGROUND: Limited data exist on the effectiveness of ceftriaxone plus doxycycline in the treatment of patients hospitalized with community-acquired pneumonia (CAP).

METHODS: We performed a retrospective cohort study of all adults hospitalized for pneumonia between January 1999 and July 2001 at an academic medical center. Outcomes were compared for patients with CAP treated with ceftriaxone plus doxycycline versus other appropriate initial empiric antibiotic therapies. Outcomes were adjusted with the use of a propensity score to account for differences in patient characteristics and illness severity between groups.

RESULTS: A total of 216 patients were treated with ceftriaxone plus doxycycline and 125 received other appropriate initial empiric antibiotic therapies. After adjustment, use of ceftriaxone plus doxycycline was associated with reduced inpatient mortality $(\mathrm{OR}=0.26,95 \% \mathrm{CI}: 0.08-0.81)$ and 30 -day mortality $(\mathrm{OR}=0.37$, 95\% CI: 0.17-0.81), but not with length of stay or readmission rates. Analysis of a subset of the sample that excluded patients admitted from nursing homes, patients admitted to the ICU, and patients diagnosed with aspiration also showed reduced inpatient mortality with the use of ceftriaxone plus doxycycline.

CONCLUSIONS: The use of ceftriaxone plus doxycycline as an initial empiric therapy for patients hospitalized with CAP appears safe and effective, and its potential superiority should be evaluated prospectively. Journal of Hospital Medicine 2006; 1:7-12. (C) 2006 Society of Hospital Medicine.

KEYWORDS: community-acquired pneumonia, doxycycline, guidelines, inpatients.

$\mathrm{n}$ the United States, community-acquired pneumonia (CAP) leads to nearly 1 million hospitalizations annually, with aggregate costs of hospitalization approaching $\$ 9$ billion. ${ }^{1,2}$ In an effort to improve the appropriate, cost-effective care for patients with CAP, several professional societies have developed clinical practice guidelines and pathways for pneumonia. ${ }^{3-7}$ Although the guidelines address all aspects of care, they devote substantial attention to antibiotic recommendations. Most U.S. guidelines recommend treatment of hospitalized patients with an intravenous beta-lactam combined with a macrolide, or a fluoroquinolone with activity against Streptococcus pneumoniae. Of the major U.S. CAP practice guidelines, only one ${ }^{6}$ recommends doxycycline as an alternative to a macrolide for inpatients.

Doxycycline is an attractive alternative to macrolides. Similar to macrolides, doxycycline is active against a wide variety of organisms including atypical bacteria (Chlamydia pneumoniae, Legionella pneumophilus, and Mycoplasma pneumoniae) and is well 
tolerated.$^{8-10}$ In addition, it is inexpensive (cost of $\$ 1.00 /$ day [awp] for $100 \mathrm{mg}$ p.o. bid), and rates of tetracycline/doxycycline resistance among S. pneumoniae isolates have remained low, in contrast to the increasing rates of resistance to macrolides and fluoroquinolones. ${ }^{11,12}$ The most recent guidelines from the Infectious Diseases Society of America cited limited published clinical data on the effectiveness of doxycycline in CAP as a barrier to increased use. ${ }^{7}$ Only one study of hospitalized patients has been published in the era of penicillinresistant pneumococcus, and this study included only 43 low-risk patients treated with doxycycline. ${ }^{13}$ At the university hospital affiliated with the University of California, San Francisco, ceftriaxone plus doxycycline is generally recommended as initial empiric antibiotic therapy for patients hospitalized with CAP, but significant variability in prescribing exists, allowing for comparisons between patients treated with different initial empiric antibiotic regimens. We compared outcomes of hospitalized patients with CAP treated with ceftriaxone plus doxycycline to those of patients treated with alternative initial empiric therapy at an academic medical center.

\section{METHODS}

\section{Study Population}

A retrospective cohort study of all adults (age $\geq 18$ years) discharged from the inpatient general medicine service of Moffitt-Long Hospital at the University of California, San Francisco, was conducted from January 1999 through July 2001. Eligibility criteria included a principal discharge diagnosis of CAP and a chest radiograph demonstrating an infiltrate within 48 hours of admission. Exclusion criteria included infection with the human immunodeficiency virus, history of organ transplantation or use of immunosuppressive therapy (including prednisone $>15 \mathrm{mg} /$ day), cystic fibrosis, postobstructive pneumonia, active tuberculosis, recent hospitalization (within 10 days), or admission for comfort care. The study protocol and procedures were reviewed and approved by the UCSF Committee for Human Research.

\section{Data Collection}

Medical record review by trained research assistants blinded to the research question was used to gather demographic data, comorbid illnesses, physical examination findings on initial presentation, and laboratory or radiographic results on initial presentation. The pneumonia severity index (PSI) score was calculated for each patient using the above data. ${ }^{14}$ In addition, data were collected on antibiotic allergies, antibiotics used within the 30 days prior to admission, results of sputum or blood cultures, and admission location (intensive care unit [ICU] versus medical floor).

Data from TSI (Transition Systems Inc., Boston, MA), the hospital administrative database, were used to identify the initial empiric antibiotic regimen. All antibiotics prescribed within the first 48 hours of hospitalization were considered initial empiric therapy with few exceptions. Initial empiric therapy was classified as 1) ceftriaxone plus doxycycline (including patients treated with these agents alone in the first 48 hours, as well as patients treated with both agents in the first 24 hours who were switched to alternative therapy [broader coverage] on the second day), or 2) other appropriate therapy (treatment consistent with current national guideline recommendations including at least a betalactam plus a macrolide or a beta-lactam plus a fluoroquinolone, or fluoroquinolone monotherapy). Patients receiving therapy inconsistent with current national guideline recommendations were excluded.

\section{Outcomes}

TSI data were used to identify length of stay, death during the index hospitalization, and return to the emergency department or readmission within 30 days of discharge. The National Death Index was used to identify all deaths that occurred after hospital discharge. The 30-day mortality data included deaths occurring during the index hospitalization and in the 30 days after the index hospitalization discharge.

\section{Statistical Analysis}

For the purposes of this analysis we compared patients treated with ceftriaxone plus doxycycline to patients treated with other appropriate therapy. To examine demographic and clinical differences between the two groups, statistical tests of comparison were performed using chi-square tests for the dichotomous variables and $t$ tests for the numeric variables, all of which were normally distributed (after log transformation in the case of length of stay).

To adjust for clinical variables that might contribute to differences in outcomes between the two groups, we used backward stepwise logistic regression analysis to construct a propensity $\operatorname{score}^{15}$ for 
the likelihood of ceftriaxone plus doxycycline use. The propensity score reflected the conditional probability of exposure to ceftriaxone plus doxycycline and allowed for stratification and, subsequently, comparisons by quintiles of propensity score. Propensity scores often have distinct advantages over direct adjustment for a large number of confounding variables and allow direct comparisons between groups with a similar propensity for receiving ceftriaxone plus doxycycline. ${ }^{15}$ Unlike random assignment of treatment, however, the propensity score cannot balance unmeasured variables that may affect treatment assignment. Thus, the possibility of bias remains. The variables used to build the score included age, presence of comorbid illness, admission from a nursing home or longterm care facility, antibiotic allergy, prior antibiotic use, PSI score, PSI risk class, diagnosis of aspiration, admission to the ICU, and positive blood cultures. The propensity score was then stratified and used as an adjustment variable in comparisons between groups for in-hospital mortality, 30-day mortality, and 30-day readmission rates. As expected, length of stay was highly skewed and was therefore logtransformed and compared between groups with adjustment for the propensity score.

To further address issues related to potential selection bias, a separate analysis was performed on a subset of the original cohort that excluded patients for whom ceftriaxone plus doxycycline would not generally be recommended as first-line therapy. For this analysis, patients admitted from a nursing home or long-term care facility, patients admitted to the ICU, and patients with a principal diagnosis of aspiration pneumonia were excluded. A propensity score was rederived for this subset, which was used to adjust for differences in outcomes. All statistical procedures were performed using STATA (Ver. 7.0, Stata Corporation, College Station TX).

\section{RESULTS}

\section{Patient Characteristics}

A total of 341 patients were eligible for analysis. Of this group, 216 were treated with ceftriaxone plus doxycycline and 125 received other appropriate therapy. Both groups of patients were similar in age. Patients treated with ceftriaxone plus doxycycline had a lower median PSI score and fewer comorbid illnesses than did patients treated with other appropriate therapy (Table 1). Blood cultures were positive in $30(8.8 \%)$ of the 341 patients in-
TABLE 1

Characteristics of Study Population

\begin{tabular}{|c|c|c|}
\hline & Ceftriaxone/doxycycline & $\begin{array}{l}\text { Other appropriate } \\
\text { therapy }\end{array}$ \\
\hline Patients $(n)$ & 216 & 125 \\
\hline Age (median) & 76 & 74 \\
\hline PSI Score (median) ${ }^{\mathrm{a}}$ & 97 & 108 \\
\hline \multicolumn{3}{|l|}{ PSI Risk Class (\%) ${ }^{a}$} \\
\hline Class I & 9.3 & 5.6 \\
\hline Class II & 11.1 & 8.8 \\
\hline Class III & 21.8 & 13.6 \\
\hline Class IV & 40.7 & 40.0 \\
\hline Class V & 17.1 & 32.0 \\
\hline Comorbid Illness (\%) & 36.1 & 47.2 \\
\hline \multicolumn{3}{|l|}{ Nursing Home/LCF } \\
\hline$(\%)^{\mathrm{a}}$ & 5.1 & 14.4 \\
\hline Aspiration (\%) ${ }^{\mathrm{a}}$ & 3.2 & 20.0 \\
\hline Admission to ICU (\%) ${ }^{\mathrm{a}}$ & 6.0 & 28.0 \\
\hline
\end{tabular}

Abbreviations: PSI, pneumonia severity index; LCF, long-term-care facility; ICU, intensive care unit. asignificant difference between groups $(P<.05)$.

cluded in the analysis, with S. pneumoniae the most commonly isolated organism ( $\mathrm{n}=17,5.0 \%)$. Of $S$. pneumoniae isolates, 4 (24\%) were resistant to penicillin (MIC $\geq 1 \mu \mathrm{g} / \mathrm{mL}$ ), and $2(12 \%)$ were resistant to tetracycline (MIC $\geq 8 \mu \mathrm{g} / \mathrm{mL}$ ).

Common antibiotic choices in patients receiving other appropriate therapy included a beta-lactam/beta-lactamase inhibitor plus doxycycline or a macrolide ( $\mathrm{n}=36,29 \%$ ), fluoroquinolone monotherapy ( $\mathrm{n}=16,13 \%)$, and a variety of other antibiotic combinations with activity against $S$. pneumoniae and atypical bacteria $(\mathrm{n}=52,42 \%)$.

\section{Clinical Outcomes}

Analyses of unadjusted outcomes showed that patients treated with ceftriaxone plus doxycycline had significantly lower inpatient ( $2 \%$ vs. $14 \%, P<.001$ ) and 30 -day ( $6 \%$ vs. $20 \%, P<.001$ ) mortality compared to patients treated with other regimens (Table 2). Multivariable logistic regression analysis identified three variables (diagnosis of congestive heart failure, admission to the ICU, and the presence of comorbid illness) associated with initial antibiotic selection, which were used to build a propensity score. After adjustment for the propensity score, use of ceftriaxone plus doxycycline remained significantly associated with lower inpatient mortality $(\mathrm{OR}=0.26,95 \% \mathrm{CI}: 0.08-0.81)$ and 30-day mortality (OR $=0.37,95 \%$ CI: $0.17-0.81$ ). Differences in length of stay and 30-day readmis- 
TABLE 2

Association between Ceftriaxone plus Doxycycline Therapy and Clinical Outcomes

\begin{tabular}{|c|c|c|c|}
\hline & $\begin{array}{l}\text { Ceftriaxone }+ \text { doxycycline } \\
(\mathrm{n}=216)\end{array}$ & $\begin{array}{l}\text { Other appropriate therapy } \\
(\mathrm{n}=125)\end{array}$ & $\begin{array}{l}\text { Adjusted odds ratio ( } 95 \% \\
\text { confidence interval) }\end{array}$ \\
\hline \multicolumn{4}{|l|}{ Inpatient } \\
\hline Mortality & $2.3 \%$ & $14.4 \%$ & $0.26(0.08-0.81)$ \\
\hline \multicolumn{4}{|l|}{ 30-day } \\
\hline mortality & $6.0 \%$ & $20.0 \%$ & $0.37(0.17-0.81)$ \\
\hline \multicolumn{4}{|l|}{$\begin{array}{l}\text { Length of stay } \\
\text { (median }\end{array}$} \\
\hline days) & 3.0 & 4.0 & $-0.09(-0.25-0.06)^{\mathrm{a}}$ \\
\hline \multicolumn{4}{|l|}{ 30-day } \\
\hline readmission & $10.7 \%$ & $12.0 \%$ & $0.87(0.42-1.81)$ \\
\hline
\end{tabular}

Propensity score adjustment was based on the variables diagnosis of congestive heart failure, admission to the intensive care unit, and presence of comorbid illness

astimated difference for the natural log of length of stay.

TABLE 3

Association between Ceftriaxone plus Doxycycline Therapy and Clinical Outcomes after Excluding Patients Admitted from Nursing Homes, Diagnosed with Aspiration, or Admitted to the ICU

\begin{tabular}{|c|c|c|c|}
\hline & $\begin{array}{l}\text { Ceftriaxone }+ \text { doxycycline } \\
(\mathrm{n}=188)\end{array}$ & $\begin{array}{l}\text { Other appropriate therapy } \\
(\mathrm{n}=70)\end{array}$ & $\begin{array}{l}\text { Adjusted odds ratio } \\
(95 \% \mathrm{CI})\end{array}$ \\
\hline Age (median years) & 75 & 71 & \\
\hline PSI score (mean) & 95 & 98 & \\
\hline \multicolumn{4}{|l|}{ Comorbid illness } \\
\hline$(\%)^{\mathrm{a}}$ & 33.5 & 50.0 & \\
\hline Inpatient mortality & $1.6 \%$ & $7.1 \%$ & $0.17(0.04-0.77)$ \\
\hline 30-day mortality & $4.8 \%$ & $8.6 \%$ & $0.43(0.14-1.31)$ \\
\hline LOS (median days) & 3 & 3 & $-0.06(-0.24-0.12)^{\mathrm{b}}$ \\
\hline \multicolumn{4}{|l|}{ 30-day } \\
\hline readmission & $11.9 \%$ & $10.0 \%$ & $1.31(0.52-3.28)$ \\
\hline
\end{tabular}

sion rates between the treatment groups were not significant (Table 2).

\section{Subset Analysis}

To address issues related to selection bias, we performed an analysis of a subset of the patients after excluding those admitted from a nursing home, diagnosed with aspiration, or admitted to the ICU, for whom ceftriaxone plus doxycycline would not be considered recommended (or "first-line") therapy. The two resulting groups were similar, except there were fewer patients with comorbid illness in the ceftriaxone plus doxycycline group $(34 \%$ vs. $50 \%, P=.015)$. The propensity score was rederived for this subset and used for adjustment. Unadjusted and adjusted outcomes are shown in Table 3. Use of ceftriaxone plus doxycycline in this subset also was associated with reduced odds of inpatient mortality $(\mathrm{OR}=0.17,95 \%$ CI: 0.04-0.77). The odds of 30-day mortality also were reduced but not significantly, as the confidence interval included $1.0(\mathrm{OR}=0.43$, 95\% CI: $0.14-1.31$ ). There were no differences between groups in length of stay or in 30-day readmission rate.

\section{DISCUSSION}

In our hospital setting, the use of ceftriaxone plus doxycycline as the initial empiric antibiotic therapy for patients hospitalized with community-acquired pneumonia was associated with significantly lower inpatient and 30-day mortality, even after adjusting for clinical differences between groups. We did not 
find a difference between regimens in hospital length of stay or 30-day readmission rate. In case the multivariable model was insufficient to account for the clinical differences (i.e., selection bias) between groups, we also performed an analysis of a subgroup of less severely ill patients by excluding those admitted from nursing homes, those admitted to the intensive care unit, and those with aspiration pneumonia. In this subset, use of ceftriaxone plus doxycycline remained associated with lower inpatient mortality but not with lower 30-day mortality. Although, as an observational study, the results of our findings could still be a result of residual confounding, we believe the results provide valuable information regarding doxycycline.

Combination therapy with a macrolide, but not doxycycline, is advocated by the practice guidelines of several major U.S. professional societies, ${ }^{3,4,7}$ apparently because of a lack of data on the effectiveness of combination therapy with doxycycline. ${ }^{7}$ Only one randomized, unblinded study, in 87 lowrisk patients hospitalized with $\mathrm{CAP}$, that compared monotherapy with IV doxycycline versus physiciandetermined therapy has been conducted. ${ }^{13}$ This study found no differences between treatment groups in clinical outcomes but did find that use of doxycycline was associated with shorter hospital stays and reduced costs. Our results, achieved in a real-world setting in relatively ill hospitalized patients ( $58 \%$ were in PSI risk class IV or V), provide further support for the use of combination therapy with doxycycline.

Hospitalized patients treated with a beta-lactam in combination with a macrolide are often discharged on macrolide monotherapy. In our population most patients treated with ceftriaxone plus doxycycline were discharged on doxycycline if they required continued therapy (data not shown). In the current era of resistance of Streptococcus pneumoniae to antibiotics, there is good reason to believe doxycycline may perform as well, if not better, than macrolides when hospitalized patients with CAP are discharged on oral monotherapy. Macrolide resistance rates among invasive pneumococcal isolates in the United States doubled from $10 \%$ to $20 \%$ during a period in which prescriptions for macrolides increased by $13 \% .{ }^{12}$ In addition, a large surveillance study of more than 1500 isolates collected in 1999 and 2000 found that $26 \%$ of the isolates were resistant to macrolides, whereas only $16 \%$ were resistant to tetracycline. ${ }^{16}$ In vitro testing against S. Pneumoniae has also suggested that tet- racycline resistance overestimates doxycycline resistance. ${ }^{17,18}$ More recently, Streptococcus pneumoniae susceptibility data from the SENTRY Antimicrobial Surveillance program reaffirmed doxycycline's in vitro superiority over macrolides. ${ }^{17}$

Our study had several limitations. The study design adopted precluded determining whether favorable results with the use of ceftriaxone plus doxycycline resulted from an effect unique to this combination of antibiotics, the possible anti-inflammatory properties of doxycycline alone, ${ }^{19,20}$ or unmeasured confounders. For example, processes of care that affect clinical outcomes for patients hospitalized with CAP, such as the timing of antibiotic delivery, the timing of blood cultures, and stability assessment on discharge were not measured in this study. To affect outcomes, these processes of care would need to be differentially distributed between our comparison groups. However, because this study was performed in a single institution during a single interval, it is likely that the performance of these processes of care would be similar for all patients.

In conclusion, ceftriaxone plus doxycycline appears to be an effective, and possibly superior, therapy for patients hospitalized with CAP. Randomized controlled trials of doxycycline-containing regimens versus other regimens are warranted.

Address for correspondence and reprint requests: Scott A. Flanders, MD, University of Michigan, 3119F Taubman Center, Box 0376, 1500 E. Medical Center Drive, Ann Arbor, Ml 48109; Fax: (734) 615-8401; E-mail: flanders@umich.edu

Received 4 September 2005; revision received 27 0ctober 2005; accepted 17 November 2005.

\section{REFERENCES}

1. Lave JR, Fine MJ, Sankey SS, et al. Hospitalized pneumonia. Outcomes, treatment patterns, and costs in urban and rural areas. J Gen Intern Med. 1996;11:415-421.

2. Niederman MS, McCombs JS, Unger AN, et al. The cost of treating community-acquired pneumonia. Clin Ther. 1998; 20:820-837.

3. Bartlett JG, Dowell SF, Mandell LA, et al. Practice guidelines for the management of community-acquired pneumonia in adults. Infectious Diseases Society of America. Clin Infect Dis. 2000;31:347-382.

4. Heffelfinger JD, Dowell SF, Jorgensen JH, et al. Management of community-acquired pneumonia in the era of pneumococcal resistance: a report from the Drug-Resistant Streptococcus pneumoniae Therapeutic Working Group. Arch Intern Med. 2000;160:1399-1408. 
5. Mandell LA, Marrie TJ, Grossman RF, et al. Canadian guidelines for the initial management of community-acquired pneumonia: an evidence-based update by the Canadian Infectious Diseases Society and the Canadian Thoracic Society. The Canadian Community-Acquired Pneumonia Working Group. Clin Infect Dis. 2000;31:383-421.

6. Niederman MS, Mandell LA, Anzueto A, et al. Guidelines for the management of adults with community-acquired pneumonia. Diagnosis, assessment of severity, antimicrobial therapy, and prevention. Am J Respir Crit Care Med. 2001; 163:1730-1754.

7. Mandell LA, Bartlett JG, Dowell SF, et al. Update of practice guidelines for the management of community-acquired pneumonia in immunocompetent adults. Clin Infect Dis. 2003;37:1405-1433

8. Cunha BA, Sibley CM, Ristuccia AM. Doxycycline. Ther Drug Monit 1982;4:115-135

9. Francke EL, Neu HC. Chloramphenicol and tetracyclines. Med Clin North Am. 1987;71:1155-1168

10. Klein NC, Cunha BA. Tetracyclines. Med Clin North Am. 1995;79:789-801

11. Neuhauser MM, Weinstein RA, Rydman R, et al. Antibiotic resistance among gram-negative bacilli in US intensive care units: implications for fluoroquinolone use. JAMA. 2003;289: 885-888

12. Hyde TB, Gay K, Stephens DS, et al. Macrolide resistance among invasive Streptococcus pneumoniae isolates. JAMA. 2001;286:1857-1862.
13. Ailani RK, Agastya G, Mukunda BN, et al. Doxycycline is a costeffective therapy for hospitalized patients with community-acquired pneumonia. Arch Intern Med. 1999;159:266-270.

14. Fine MJ, Auble TE, Yealy DM, et al. A prediction rule to identify low-risk patients with community-acquired pneumonia. N Engl J Med. 1997;336:243-250.

15. Rosenbaum PR RD. Reducing bias in observational studies using subclassification on the propensity score. J Am Stat Assoc. 1984; 79:516-524

16. Doern GV, Heilmann KP, Huynh HK, et al. Antimicrobial resistance among clinical isolates of Streptococcus pneumoniae in the United States during 1999 - 2000, including a comparison of resistance rates since 1994-1995. Antimicrob Agents Chemother. 2001;45:1721-1729

17. Jones RN, Sader HS, Fritsche TR. Doxycycline use for community-acquired pneumonia: contemporary in vitro spectrum of activity against Streptococcus pneumoniae (1999-2002). Diagn Microbiol Infect Dis. 2004;49:147-149

18. Shea KW, Cunha BA, Ueno Y, et al. Doxycycline activity against Streptococcus pneumoniae. Chest. 1995; 108:17751776.

19. Pruzanski W, Greenwald RA, Street IP, et al. Inhibition of enzymatic activity of phospholipases A2 by minocycline and doxycycline. Biochem Pharmacol. 1992;44:1165-1170.

20. D'Agostino P, La Rosa M, Barbera C, et al. Doxycycline reduces mortality to lethal endotoxemia by reducing nitric oxide synthesis via an interleukin-10-independent mechanism. J Infect Dis. 1998;177:489-492. 